\title{
Comprehensive evaluation of the response of genes to the administration of the antitumor drug S-1 using a low density array
}

\author{
HISASHI MATSUOKA ${ }^{1}$, KAZUYA KONDO ${ }^{2}$, HIROMITSU TAKIZAWA ${ }^{1}$, HARUHIKO FUJINO $^{1}$, \\ ETSUKO SAKAMOTO $^{3}$, JUNJI UCHIDA ${ }^{4}$, KOH UYAMA ${ }^{1}$, HIROAKI TOBA ${ }^{1}$, \\ KOICHIRO KENZAKI ${ }^{1}$, SHOJI SAKIYAMA ${ }^{1}$ and AKIRA TANGOKU ${ }^{1}$

\begin{abstract}
Departments of ${ }^{1}$ Thoracic, Endocrine Surgery and Oncology, and ${ }^{2}$ Oncological Medical Services, Institute of Health Biosciences, The University of Tokushima Graduate School, Tokushima 770-8509; ${ }^{3}$ Tsukuba Research Center, Taiho Pharmaceutical Co., Ltd., Tsukuba;

${ }^{4}$ Tokushima Research Center, Taiho Pharmaceutical Co., Ltd., Tokushima, Japan
\end{abstract}

Received August 25, 2014; Accepted October 9, 2014

DOI: $10.3892 /$ ijo.2014.2754

\begin{abstract}
S-1 is a newly developed dihydropyrimidine dehydrogenase inhibitory fluoropyrimidine that exhibits high clinical efficacy against non-small cell lung cancers. To identify genes that may be associated with chemosensitivity to the antitumor drug S-1, we used a low density array representing 93 genes to analyze expression profiles in 4 orthotopically implanted lung cancers derived from human lung cancer cell lines (Lu99, Lu130, LC6 and A549). The tumor growth inhibition (TGI) rates of S-1 in orthotopically implanted tumors of the Lu99, Lu130, LC6 and A549 cell lines were 34.6, 37.5, 32.1 and 3.6\%, respectively. The expression of the PRSS3, ABCC4, TXN, SHMT1 and CMPK genes was significantly promoted in the orthotopically implanted SCID mouse model of the 4 lung cancer cell lines by the administration of S-1, while the expression of the LMO7 and FOLH1 genes was significantly suppressed. The expression of the ABCC1, 2 and TST genes was negatively correlated with TGI. The expression of the TK1 and ERCC2 genes was positively correlated with TGI. The results of the present study suggest that the expression of the ABCC1, 2, TST, TK1 and ERCC2 genes is related to resistance to the antitumor drug S-1.
\end{abstract}

\section{Introduction}

S-1 is a newly developed DIF [DPD (dihydropyrimidine dehydrogenase: both an initial and rate-limiting catabolic enzyme

Correspondence to: Professor Kazuya Kondo, Department of Oncological Medical Services, Institute of Health Biosciences, The University of Tokushima Graduate School, 18-15 Kuramoto-cho 3, Tokushima 770-8509, Japan

E-mail: kondo@clin.med.tokushima-u.ac.jp

Key words: anticancer drug S-1, non-small cell lung cancer, orthotopic animal model, low density array, dihydropyrimidine dehydrogenase inhibitory fluoropyrimidine, drug resistance of 5-FU) inhibitory fluoropyrimidines] that consists of $1 \mathrm{M}$ tegafur, 0.4 M gimeracil (a potent DPD inhibitor), and $1 \mathrm{M}$ oteracil (an oratate phosphoribosyltransferase inhibitor) to protect against gastrointestinal toxicity. Several studies have comprehensively examined genes that predict the response of cancer cells to chemotherapies, including 5-FU, using in vitro cancer cell lines and xenograft subcutaneous implantation models (1-4).

Preclinical tumor models are very important in determining the study and design of new regimens for cancer treatments. Considerable shortcomings have been reported in ectopic subcutaneously implanted models (xenograft models) (5). Paget proposed the original 'seed and soil' theory in 1889, in which the organ-site-specific implantation of tumor cells was considered essential for the optimal growth and progression of tumors in vivo (6). Tumors in xenograft models generally grow rapidly and do not mimic the markedly slower doubling times of most human cancers, which may render them more sensitive to most chemotherapy drugs than target-dividing cells. Tumors in xenograft models are often treated with drugs at doses or pharmacokinetics that are not relevant to humans. The metastatic patterns of tumors in xenograft models differ from those of tumors in clinics. On the other hand, the possibility of distant metastatic spread is higher with the orthotopic transplantation of tumors than with subcutaneous transplants. Orthotopic tumor models have established metastases that include the multiple sequential steps associated with the metastatic cascade. The metastatic patterns and pharmacokinetics of these models are similar to those of tumors in clinics. We established a new, patient-like model of lung cancer metastasis by orthotopic implantation using human non-small cell lung cancer cell lines. Regardless of the administration routes (oral or intraperitoneal) and characteristics of anticancer drugs, cytostatic or cytotoxic, our model was capable of evaluating the inhibitory effect of anticancer drugs (7).

To identify genes that may be associated with chemosensitivity to the antitumor drug S-1, we used a low density array representing 93 genes to analyze expression profiles in 
4 orthotopically implanted lung cancers derived from human lung cancer cell lines.

\section{Materials and methods}

This study was approved by The Committee for the Care and Use of Animals in The University of Tokushima Faculty of Medicine and was performed by the Institutional Animal Care and Oversight Committee according to established guidelines.

Animals. Male SCID mice (6 weeks of age) with a CB-17 genetic background were purchased from CLEA Japan, Inc. (Tokyo, Japan). These mice had been raised from birth in a specific pathogen-free environment.

Cell lines. The Lu99, Lu130, LC6, and A549 lung cancer cell lines were used in this study. Lu99 was kindly provided by the Institute of Development, Aging and Cancer, Tohoku University (Sendai, Japan). Lu130 and LC6 were obtained from the Central Institute for Experimental Animals (Kawasaki, Japan), A549 was purchased from the American Type Culture Collection (ATCC, Manassas, VA, USA). Cells were cultured in RPMI-1640 with $10 \%$ heat-inactivated fetal bovine serum (Bioscience Pty, Ltd., Australia) and maintained at $37^{\circ} \mathrm{C}$ in a humidified incubator with $5 \% \mathrm{CO}_{2}$ in air. We selected these 4 cell lines because a previous study demonstrated that these cell lines had different tumor growth inhibition (TGI) rates for S-1 in subcutaneously implanted xenograft models (Lu99, 62\%; Lu130, 31\%; LC6, 45\%; A549, 56\%) (8).

Chemicals. S-1 (1 M tegafur, 0.4 M 5-chloro-2,4-dihydroxypyridine and $1 \mathrm{M}$ potassium oxonate) was provided by Taiho Pharmaceutical Co., Ltd.

Surgical orthotopic implantation of human lung cancer cells in SCID mice (Fig. 1). As shown in our previous study (9), cells were harvested for implantation at 70-80\% confluence using $1 \mathrm{mmol} / \mathrm{l}$ EDTA (Wako Pure Chemical Industries, Ltd., Osaka, Japan) in PBS (Nissui Pharmaceutical Co., Ltd., Tokyo, Japan). Cells were washed in RPMI-1640 and resuspended to a final concentration of $2.0 \times 10^{6}$ cells/ml in RPMI-1640 containing $0.1 \%$ BSA (Boehringer-Mannheim, Mannheim, Germany). Mice were fully anesthetized by ether inhalation and placed in the right lateral decubitus position with the four limbs restrained. A 1-cm transverse incision was made on the left lateral skin just below the inferior border of the scapula of the SCID mouse. Muscles were separated from the ribs by sharp dissection, and intercostal muscles were exposed. The left lung was visible through the intercostal muscles. A 30-gauge needle was inserted $\sim 5 \mathrm{~mm}$ into the lung through the intercostal muscle, and an inoculum of $2.0 \times 10^{6}$ tumor cells $/ \mathrm{ml}$ with $400 \mu \mathrm{g} / \mathrm{ml}$ Matrigel was then dispersed into the left lung at a final volume of $10 \mu \mathrm{l}\left(2.0 \times 10^{4}\right.$ cells $)$ medium. The skin incision was closed with 3-0 silk. We examined the growth of the tumor in the lung when the animal was sacrificed, and determined mice with tumors in their left lungs to be success cases. The success rate of implantation varied according to the cell line (50-75\%).

Evaluation of the effects of $S-1$ on orthotopically implanted SCID mice. Forty orthotopic implantation models were made from the Lu99 cell line. Eight mice were sacrificed 6 weeks after the implantation and 3 tumors implanted in the lungs were obtained. These tumors were resected and their weights and volumes were measured (pre-administration group, $n=3$ ). The remaining 32 mice were allocated randomly to 2 groups: $\mathrm{S}-1$ administration group $(n=16)$ and control group $(n=16)$. Sixteen mice were administered $10 \mathrm{mg} / \mathrm{kg}$ body weight of $\mathrm{S}-1$ orally for 5 consecutive days per week for 3 weeks (S-1 group, $n=16)(8)$. The control group $(0 \mathrm{mg} / \mathrm{kg}$ body) only received $0.5 \%$ HPMC solution (control group, $\mathrm{n}=16$ ). Mice were then sacrificed. Nine of 16 tumors in the S-1 group (success rate, 56\%) and 9 of 16 tumors in the control group (success rate, 56\%) were located in the lung. These tumors were resected and their weights and volumes were measured. Forty models were made from the Lu130 cell line. Eight mice were sacrificed 6 weeks after the implantation, and 3 tumors implanted in the lungs were obtained. These tumors were resected and their weights and volumes were measured (pre-administration group, $n=3$ ). The remaining 32 mice were allocated randomly to 2 groups: S-1 administration group $(n=16)$ and control group $(n=16)$. After 3 weeks of the administration protocol, 15 of 16 tumors in the S-1 group (success rate, 94\%) and 11 of 16 tumors in the control group (success rate, 69\%) were located in the lung. Thirty-five models were made from the LC6 cell line. Seven mice were sacrificed 6 weeks after the implantation, and 3 tumors implanted in the lungs were obtained. These tumors were resected and their weights and volumes were measured (pre-administration group, $\mathrm{n}=3$ ). The remaining 28 mice were allocated randomly to 2 groups: $\mathrm{S}-1$ administration group $(n=14)$ and control group $(n=14)$. After 3 weeks of the administration protocol, 6 of 14 tumors in S-1 group (success rate, $43 \%$ ) and 9 of 16 tumors in control group (success rate, 56\%) were located in the lung. Thirty-five models were made from the A549 cell line. Five mice were sacrificed 6 weeks after the implantation, and 3 tumors implanted in the lungs were obtained. These tumors were resected and their weights and volumes were measured (pre-administration group, $n=3$ ). The remaining 30 mice were allocated randomly to 2 groups: S-1 administration group $(n=15)$ and control group $(n=15)$. After 3 weeks of the administration protocol, 7 of 15 tumors in S-1 group (success rate, 47\%) and 7 of 15 tumors in control group (success rate, $47 \%$ ) were located in the lung.

We evaluated antitumor effects and side effects. Tumor volume was calculated using the following formula: Tumor volume $\left(\mathrm{mm}^{3}\right)=$ Length $(\mathrm{mm}) \times$ Width $(\mathrm{mm})^{2} / 2$. Relative tumor volume (RTV) was calculated as follows: tumor volume in the S-1 group or control group/tumor volume in the preadministration group. The antitumor effect [inhibition rate (\%)] was calculated as follows: inhibition rate $(\%)=(1-$ mean RTV in the S-1 group/mean RTV in the control group) $x$ 100. The tumor growth inhibition rate on the last day of the administration protocol was considered to reflect the antitumor effect. The body weight of each mouse was measured twice a week to monitor the toxicity of S-1.

Measurement of gene expression using a low density array (LDA). Total RNA was isolated from tissue using the RNeasy mini kit (Qiagen, Tokyo, Japan) and reverse transcribed using a high capacity cDNA reverse transcription kit (Applied Biosystems, Foster City, CA, USA) according to the manu- 


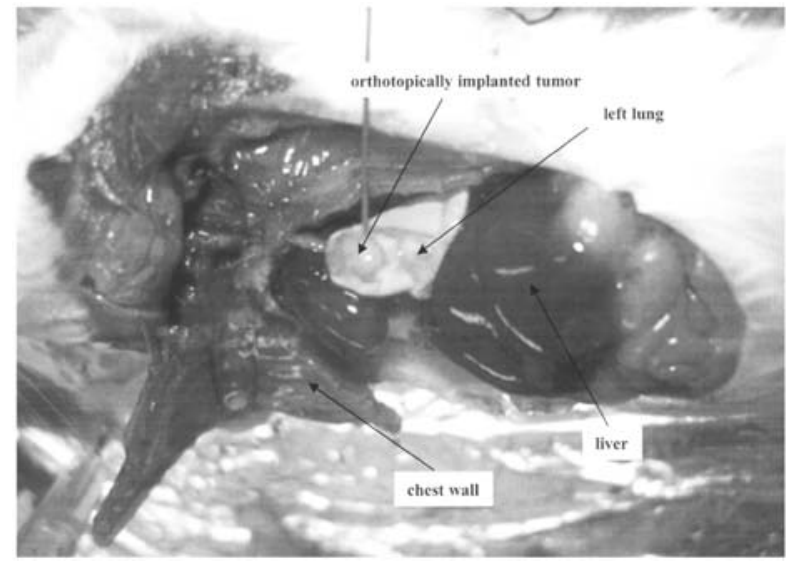

Figure 1. Orthotopically implanted tumors at sacrifice of the SCID mice. Orthotopically implanted mice were sacrificed 6 weeks after implantation and the tumor weights, tumor sizes, and dissemination and metastasis were examined.

facturer's protocol. We examined the expression of 96 genes containing enzymes involved in the metabolism of 5-FU by the LDA (Applied Biosystems) (Table I). The LDA contained 8 sample-loading lines, with each being connected by a micro-channel to 48 miniature reaction chambers for a total of 384 wells per card. Gene-specific primers and TaqMan probes were factory-designed and embedded in each well. The LDA in this study was configured into 4 identical 96-gene sets (2 samples in duplicate). Gene expression levels were normalized to the geometric mean of 3 reference genes (ACTB, GAPDH and RPLP), and $\log 2$-transformed for analysis.
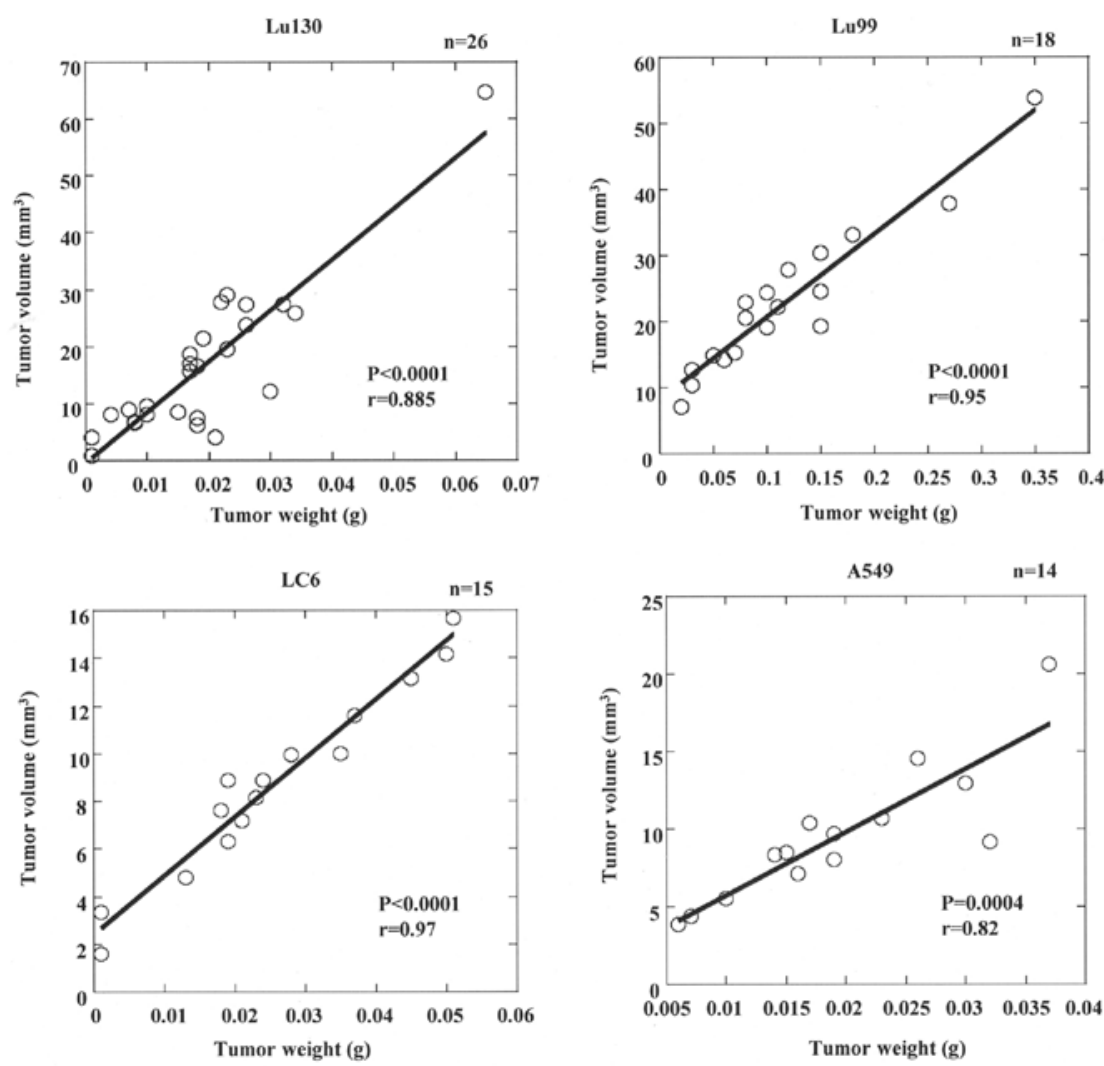

Figure 2. Relationship between tumor weight and tumor volume in orthotopically implanted tumors.
A total of $100 \mu 1$ reaction mixture with $5 \mu 1$ cDNA template (100 ng cDNA) and $50 \mu 1$ 2x TaqMan Universal PCR Master Mix (Applied Biosystems) was added to each line of the LDA after vortexing and brief centrifugation. Each reaction cell contained $1 \mu \mathrm{l}$ reaction mixture with $1 \mathrm{ng}$ cDNA. The LDA was sealed with a TaqMan low density array sealer (Applied Biosystems) before centrifugation in a Sorvall Legend ${ }^{\mathrm{TM}}$ centrifuge (Kendro Scientific, Asheville, NC, USA) for $1 \mathrm{~min} \times 2$ at 12,000 rpm. PCR amplifications were performed in the micro-fluidic card sample block of an ABI PRISM ${ }^{\circledR}$ 7900HT sequence detection system (Applied Biosystems). The amplification protocol was used as below: 2 min at $50^{\circ} \mathrm{C}$ to activate uracil-DNA glycosylase (UNG), $10 \mathrm{~min}$ at $94.5^{\circ} \mathrm{C}$ (activation), 40 cycles of denaturation at $97^{\circ} \mathrm{C}$ for $30 \mathrm{sec}$, and annealing and extension at $59.7^{\circ} \mathrm{C}$ for $1 \mathrm{~min}$. The threshold cycle, $\mathrm{Ct}$ was automatically given by the SDS2.2 software package (Applied Biosystems).

Statistical analysis. Clustering was performed using GeneSpring software (Agilent Technologies, Santa Clara, CA, USA). The Student's t-test was used to analyze differences between groups. Pearson's correlation coefficients were used to assess the association between tumor weight and tumor volume and between TGI and gene expression levels. JMP software (SAS Institute Inc., Cary, NC, USA) was used for statistical analysis. Data were considered significant at $\mathrm{P}<0.05$.

\section{Results}

Weights of orthotopically implanted tumors. Fig. 2 shows the relationship between tumor weight and tumor volume in all 
Table I. The 96 genes studied.

\begin{tabular}{|c|c|}
\hline Common name & Description \\
\hline 1 АCTB & \multirow{3}{*}{ House keeping gene } \\
\hline 2 GAPD & \\
\hline 3 RPLPO & \\
\hline 4 ATIC & \multirow{21}{*}{ Folate metabolism } \\
\hline 5 DHFR & \\
\hline 6 FPGS & \\
\hline 7 FTHFD (ALDH1L1) & \\
\hline 8 GART & \\
\hline $9 \mathrm{GGH}$ & \\
\hline 10 MTHFD1 & \\
\hline 11 MTHFR & \\
\hline 12 MTRR & \\
\hline 13 MTR & \\
\hline 14 SHMT1 & \\
\hline 15 AMT & \\
\hline 16 FOLH1 & \\
\hline 17 FOLR1 & \\
\hline 18 FOLR2 & \\
\hline 19 FOLR3 & \\
\hline 20 MFTC & \\
\hline 21 MTHFD1L & \\
\hline 22 MTHFD2 & \\
\hline 23 MTHFS & \\
\hline 24 SHMT2 & \\
\hline 25 DPYD & \multirow{18}{*}{ 5-FU metabolism } \\
\hline 26 DPYS & \\
\hline 27 DTYMK & \\
\hline 28 DUT & \\
\hline 29 ECGF1 (TP) & \\
\hline 30 RRM1 & \\
\hline 31 RRM2 & \\
\hline 32 RRM2B & \\
\hline 33 TK1 & \\
\hline 34 TYMS & \\
\hline 35 UMP-CMPK & \\
\hline 36 UMPK & \\
\hline 37 UMPS & \\
\hline 38 UPP1 & \\
\hline 39 UPP2 & \\
\hline 40 E2F1 & \\
\hline $41 \mathrm{CDA}$ & \\
\hline $42 \mathrm{DCK}$ & \\
\hline
\end{tabular}

\section{ALDH2}

44 ASL

45 DKFZp564J157

46 EPS8

47 GPX2

48 IL2ORA

49 INPP1

50 LMO7
Table I. Continued.

Common name Description

$\begin{array}{llc}51 & \text { MGAT4B } & \text { S-1 response related genes in } \\ 52 & \text { NET1 } & \text { the } 30 \text { xenografts } \\ 53 & \text { PRSS2 } & \\ 54 & \text { PRSS3 } & \\ 55 & \text { SART2 } \\ 56 & \text { SNRPF } \\ 57 & \text { TCF7L1 } \\ 58 & \text { TPBG } \\ 59 & \text { TST } & \\ 60 & \text { XPNPEP1 }\end{array}$
61 RNF130
62 SUCLG1
63 TFAP2A
$64 \mathrm{CCNH}$
65 CDC40
66 DHRS 8
67 EPB41L2

$\begin{array}{lll}68 & \text { ERCC1 } & \\ 69 & \text { ERCC2 } & \\ 70 & \text { XRCC1 } & \\ 71 & \text { GSTP1 } & \text { CDDP } \\ 72 & \text { GCLC } & \\ 73 & \text { TXN } & \\ 74 & \text { XPA } & \\ 75 & \text { DDB2 } & \end{array}$

76 ABCB 1

77 ABCC1

$78 \mathrm{ABCC} 11$

79 ABCC2

80 ABCC3

81 ABCC4

82 ABCG2

83 SLC19A1

84 SLC29A1
$85 \mathrm{EGF}$

86 ERBB2

87 ERBB3

88 ERBB4

89 IGF1R

90 IGF1

91 TGFA

92 EGFR
93 MLH1
94 MSH2
95 MSH6
96 PMS2

S-1 response related genes in the 7 lung cancer cell lines 

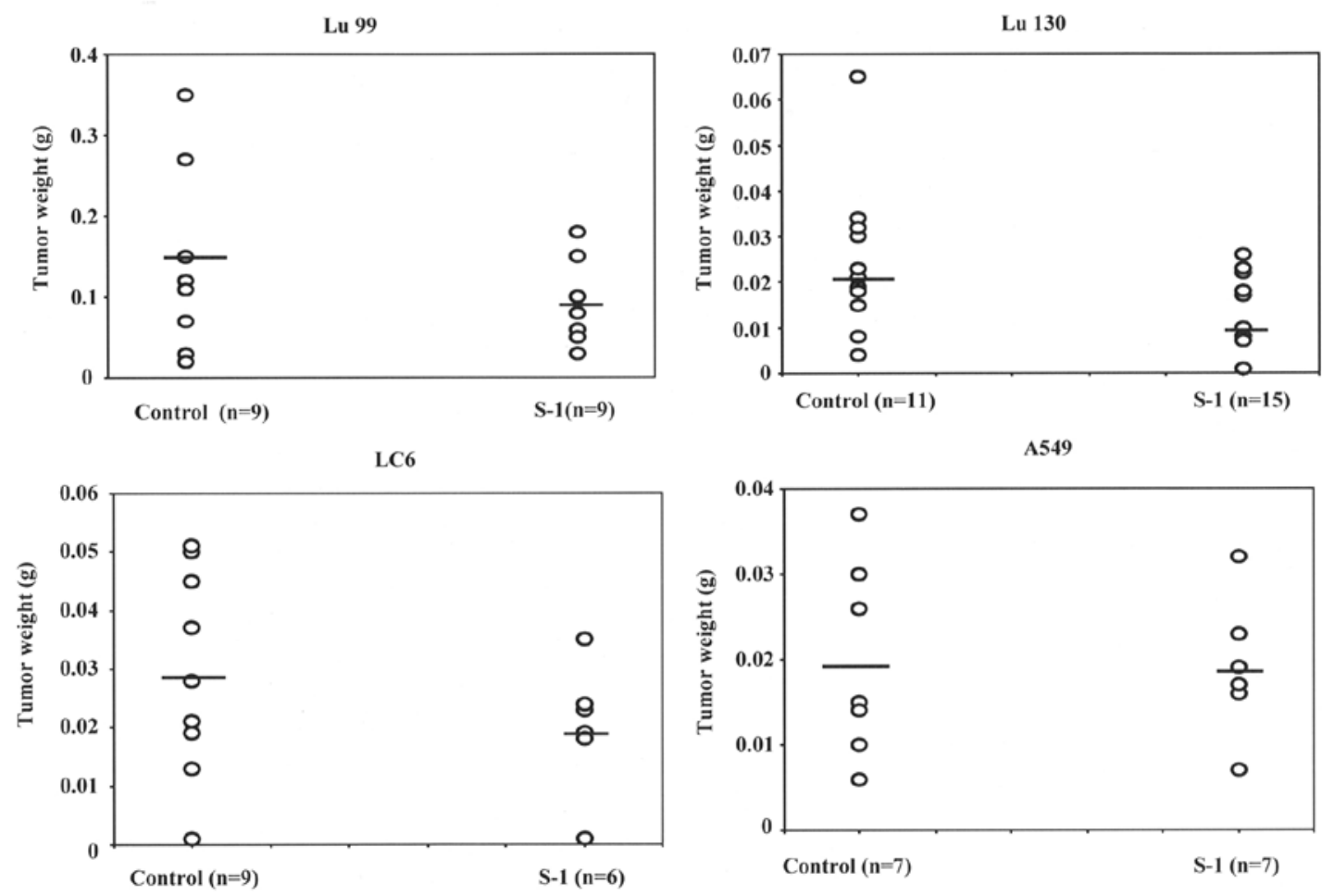

Figure 3. The weight of orthotopically implanted tumors in the control and S-1 groups in each cell line. Lu99, S-1=92 $\pm 48 \mathrm{mg}$, control=141 $\pm 108 \mathrm{mg}$; Lu130,

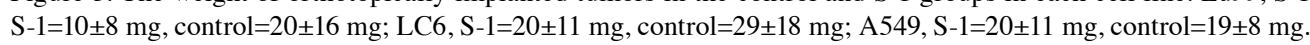

orthotopically implanted tumors that could be measured. A correlation was observed between tumor weight and tumor volume in each cell line (Lu99, Pearson's correlation coefficient, $\mathrm{r}=0.95, \mathrm{P}<0.0001$; Lu130, $\mathrm{r}=0.87, \mathrm{P}<0.0001$; LC6, $\mathrm{r}=0.97, \mathrm{P}<0.0001 ; \mathrm{A} 549, \mathrm{r}=0.82, \mathrm{P}=0.0004)$. We determined that tumor weight could be used as an indicator of the response.

Fig. 3 shows the weights of orthotopically implanted tumors in the control and S-1 groups in each cell line. In Lu99, Lu130 and LC6 cell lines, the tumor weight in the S-1 group was less than that in the control group (Lu99, S-1=92 $\pm 48 \mathrm{mg}$, vs control $=141 \pm 108 \mathrm{mg}, \mathrm{P}=0.241 ; \mathrm{Lu} 130, \mathrm{~S}-1=10 \pm 8 \mathrm{mg}$ vs control $=20 \pm 16 \mathrm{mg}, \mathrm{P}=0.093$; LC6, S-1=20 $\pm 11 \mathrm{mg}$ vs control $=29 \pm 18 \mathrm{mg}, \mathrm{P}=0.223$ ). On the other hand, the tumor weight in the S-1 group in the A549 cell line was similar to that in the control group $(\mathrm{S}-1=20 \pm 11 \mathrm{mg}$ vs control $=19 \pm 8 \mathrm{mg}$, $\mathrm{P}=0.893)$. The TGI rates of $\mathrm{S}-1$ in the orthotopically implanted tumors of the Lu99, Lu130, LC6, and A549 cell lines were $34.6,37.5,32.1$ and $3.6 \%$, respectively. The TGI rates of S-1 in orthotopically implanted tumors in Lu130 and LC6 were similar to those in subcutaneously implanted tumors (Lu130, 38 vs 31\%; LC6, 32 vs 45\%) (8). However, the TGI rates of S-1 in orthotopically implanted tumors in Lu99 and A549 were different from those in subcutaneously implanted tumors (Lu99, 35 vs 62\%; A549, 3.6 vs 56\%).

Changes in body weights in the control and S-1 groups of the Lu99 cell line are shown in Fig. 4. Body weights in the control and S-1 groups were 20.54 $\pm 3.04 \mathrm{~g}$ and $22.31 \pm 3.23 \mathrm{~g}$ on day 21 , respectively. No significant difference was observed between body weights in the control or S-1 groups. In the 3 other cell lines, no significant differences were observed between body weights in the control or S-1 groups (Lu130, control 23.81 \pm 2.05 vs S-1 23.73 \pm 1.49 ; LC6, control 24.29 \pm 2.32

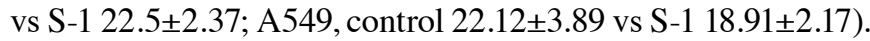

Cluster analysis of gene expression in the 4 cell lines (Lu99, Lu130, LC6, and A549) in the control group. Fig. 5 shows cluster analysis of the control group in Lu99, Lu130, LC6 and A549, and revealed a specific gene expression pattern for each cell line. We compared the pre-administration group and control group, and control group and S-1 group in each cell line, because the expression of genes was different in the 4 cell lines.

Comparison of the expression of genes between the control group and pre-administration group. To identify the genes influenced by tumor growth, we compared the expression of genes between the pre-administration group (tumors 6 weeks after the implantation) and control group (tumors 9 weeks after the implantation). A significant difference in only the MTHFD1L gene, was observed between the control group and pre-administration group in the Lu99 line. In the Lu130 line, a significant difference was observed in 3 genes, PRSS2, UPP1, and MTHFD1L, with the expression of the PRSS2 gene being 12.2-fold higher. In the LC6 line, a significant difference was observed in 9 genes, ERCC2, GPX2, LMO7, FOLH1, GART, TCF7L1, TGFA, MSH2 and ABCB1, and the expression levels of GPX2, LMO7, FOLH1 and ABCB1 were 1.50-, 1.59-, 3.42and 1.7-fold higher, respectively. In the A549 line, a significant difference was observed in TK1, DHFR, DUT, CCNH, E2F1, SHMT1, MTHFS, FOLR1 and ABCC2.

Comparison of the expression of genes in the control group and $S-1$ group. To identify genes influenced by the admin- 

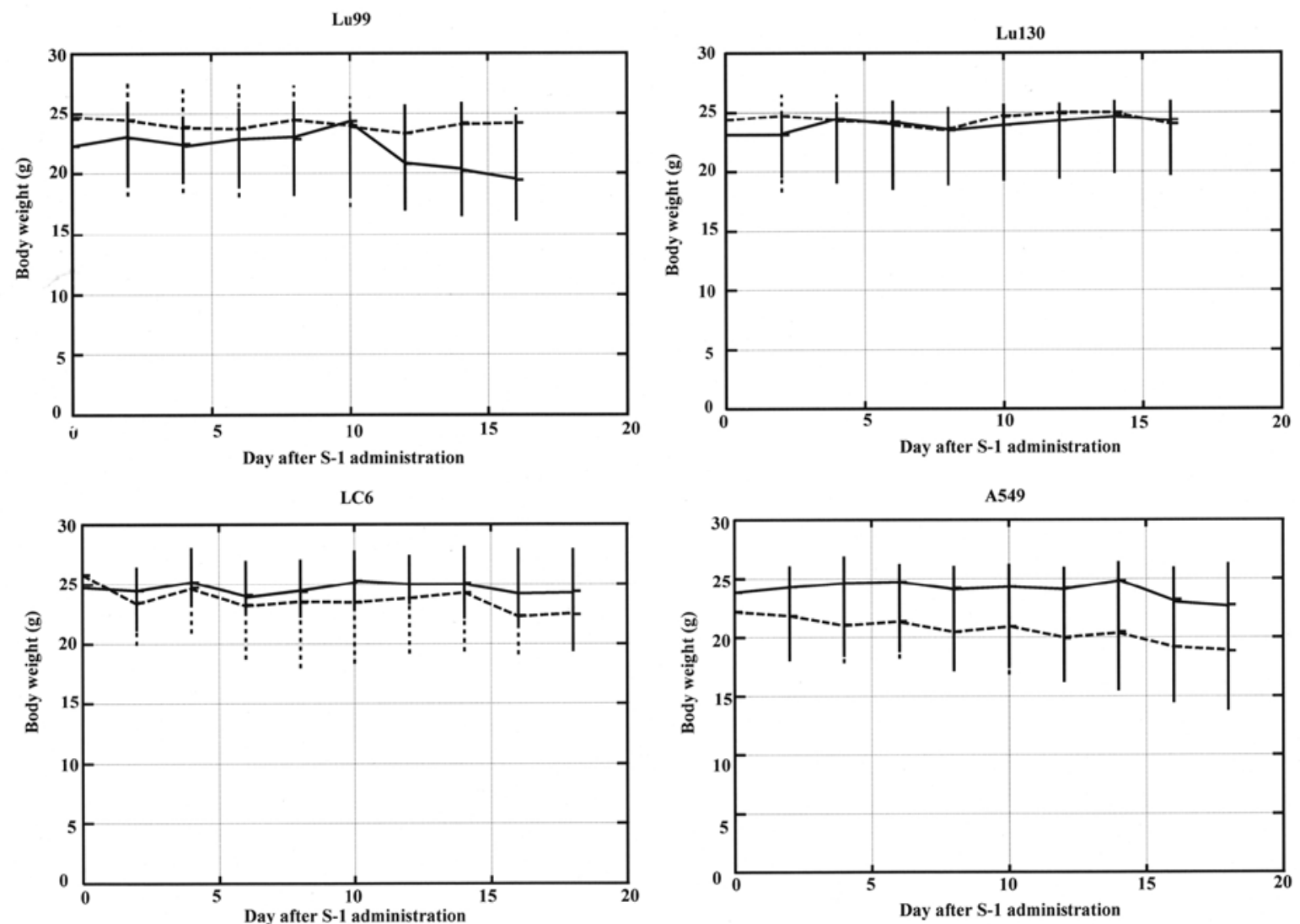

Figure 4. The body weight of orthotopically implanted mice after the administration of S-1 (S-1 group, dotted line; control group, continued line).

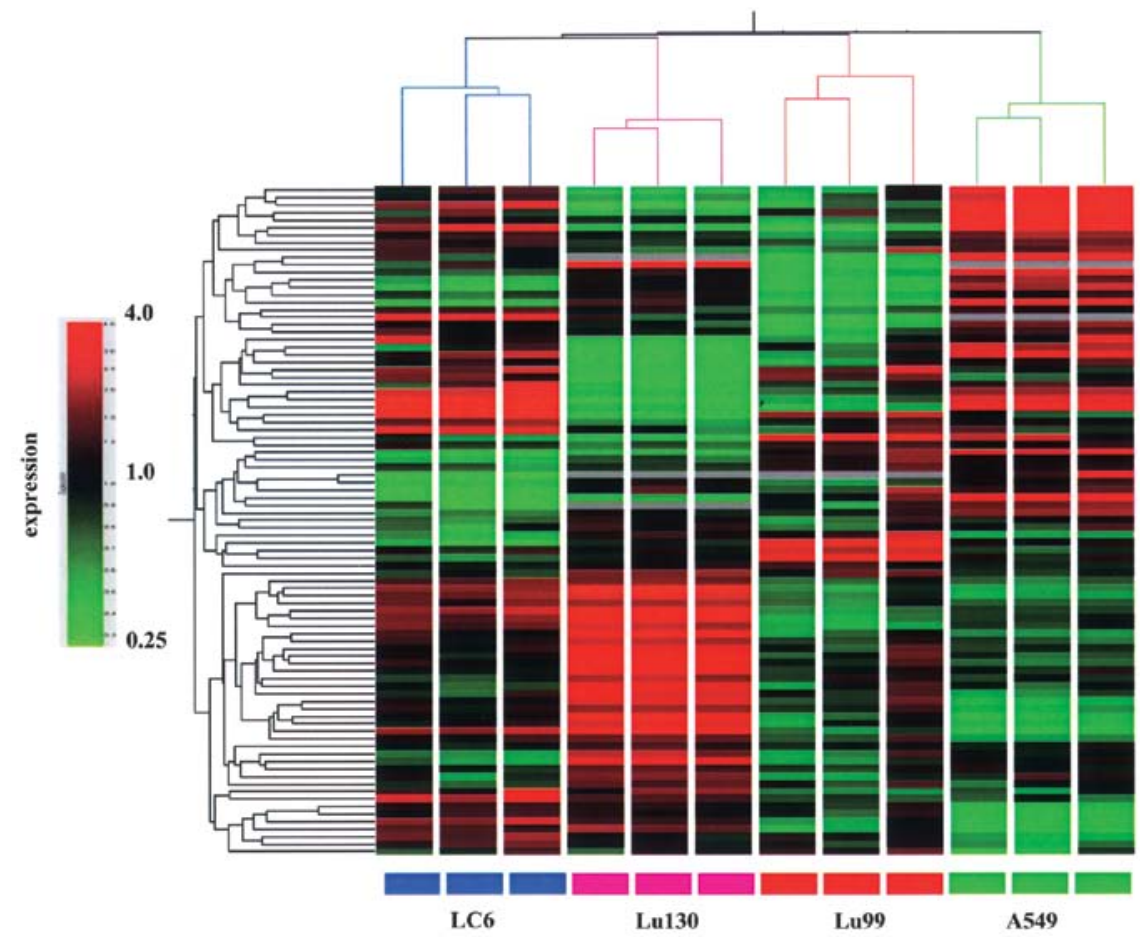

Figure 5. Cluster analysis of gene expression patterns in the control group in the Lu99, Lu130, LC6 and A549 cell lines.

istration of $\mathrm{S}-1$, we compared the expression of genes in the control group and S-1 group. In the Lu99 line, a significant difference was observed in 4 genes, PRSS3, ABCC4, TK1 and MTHFD1L, and the expression levels of the PRSS3 and ABCC4 genes were 1.7- and 1.6-fold higher, respectively. In the Lu130 line, a significant difference was observed in only 

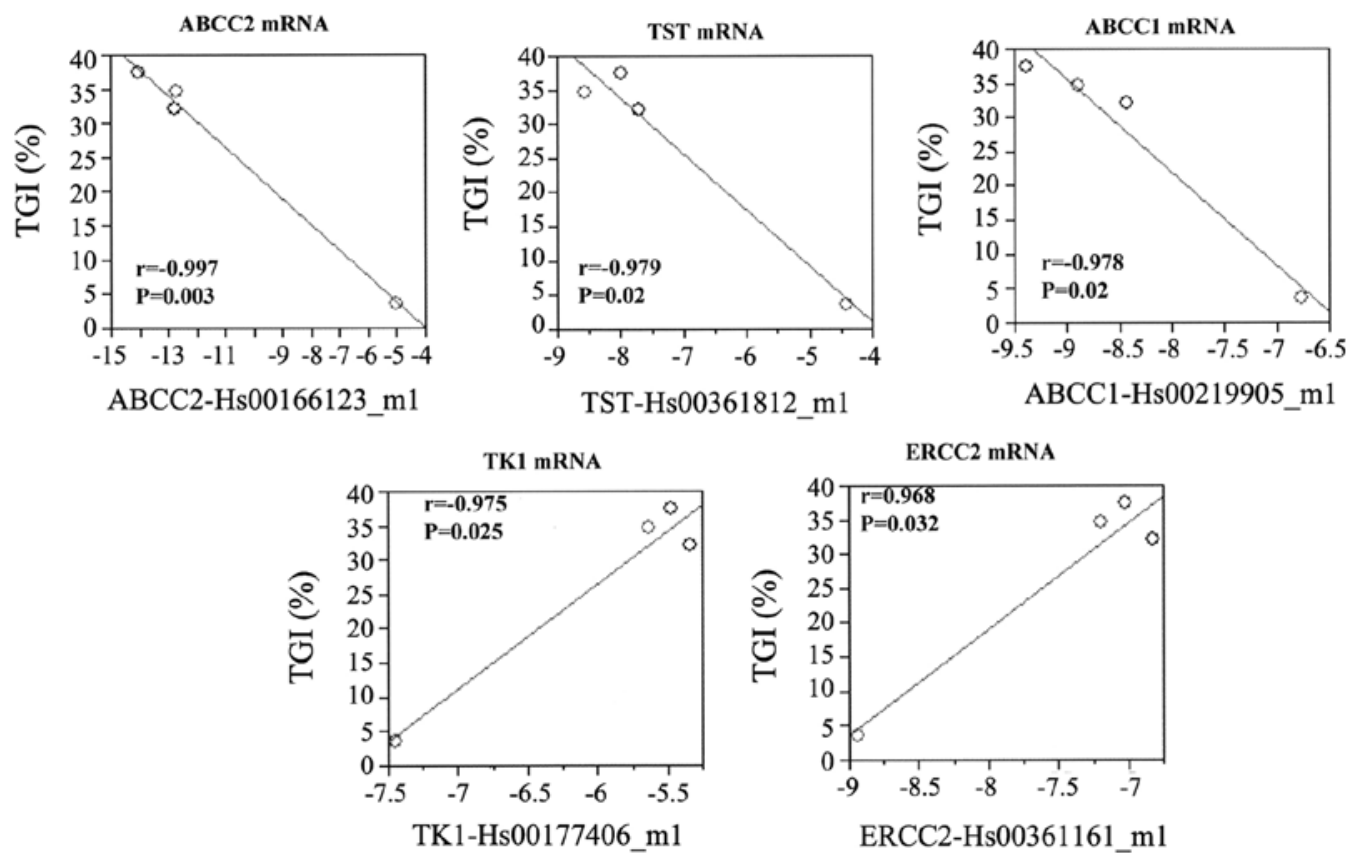

Figure 6. The correlation between gene expression and the tumor growth inhibition rate of five genes in the four cell lines.

the UPP1 gene. In the LC6 line, a significant difference was observed in 6 genes, SART2, ASL, LMO7, IL2ORA, FOLH1 and GART, and the expression levels of the LMO7 and FOLH1 genes were 0.64- and 0.24-fold lower, respectively. In the A549 line, a significant difference was observed in 6 genes, SHMT1, MTHFR, TXN, ALDH2, CMPK and ASL, and the expression levels of the SHMT1, TXN and CMPK genes were 1.54-, 2.01and 2.12-fold higher, respectively.

Correlative analysis between TGI and gene expression in the cell lines (Pearson's correlation coefficient). We performed correlative analysis between TGI and the expression of 96 genes in the 4 cell lines (Pearson's correlation coefficient). We identified 5 genes, 3 of which, ABCC2 [correlation coefficient (CC), -0.997, $\mathrm{P}=0.003$ ], TST (CC -0.979, $\mathrm{P}=0.021$ ), and ABCC1 (CC, -0.978, $\mathrm{P}=0.022$ ), were positively correlated with TGI. The 2 remaining genes, TKI $(\mathrm{CC}, 0.975, \mathrm{P}=0.025)$ and ERCC2 (CC, 0.968, $\mathrm{P}=0.032)$, were negatively correlated with TGI (Fig. 6). A correlation was observed between gene expression and TGI for the 5 genes.

\section{Discussion}

Preclinical tumor models are a fundamental component of the study and design of new regimens for cancer treatments. Our goal was to establish a method that could provide novel insights into the mechanism underlying resistance and sensitivity to the anticancer drug S-1 in preclinical tumor models that are very similar to clinical lung cancers. Although subcutaneously implanted tumor models are relatively easily generated and measurable in mice, considerable shortcomings have been reported in these models. The characteristics of tumors in subcutaneous implantation may not reflect the original tissues and may also differ from the original environments. We used orthotopic tumor models in SCID mice employing several lung cancer cell lines. In this study, the TGI rates of S-1 in orthotopically implanted tumors in Lu130 and LC6 were similar to those in subcutaneously implanted tumors (Lu130, 38 vs 31\%; LC6, 32 vs 45\%) (3). However, the TGI rates of S-1 in orthotopically implanted tumors in Lu99 and A549 were different from those in subcutaneously implanted tumors (Lu99, 35 vs 62\%; A549, 3.6 vs 56\%) (3). Cancer cell lines implanted subcutaneously in mice generally grow rapidly and do not mimic the markedly slower doubling times of most human cancers, which may render them more sensitive to most chemotherapy drugs than target-dividing cells (1). This study demonstrated that the TGI rate of S-1 in orthotopically implanted tumors was lower than that in subcutaneously implanted tumors in 3 cell lines (LC 6, Lu99 and A549).

The success rate of implantation was $50-75 \%$ in the 4 cell lines used in the present study. The implantation rate in this study was slightly lower than that in previous studies using the lung cancer cell lines Ma44-3, A549, Ma-11, FT and FM (70-90\%) (10-12). We performed implantation using the cell lines Lu130, LC6 and Lu99 for the first time in this study. The lower implantation rate may have been due to the characteristics (including growth rate, invasive features, and migration capacity) of each cell line. We used a larger number of mice in this study than in previous studies because we could not confirm the success of implantation until mice were sacrificed. We considered an implantation rate of $>50 \%$ to be acceptable.

In the orthotopically implanted SCID mouse model of the 4 lung cancer cell lines, the expression of the PRSS3, ABCC4, TXN, SHMT1 and CMPK genes was significantly induced by the administration of S-1, while the expression of the LMO7 and FOLH1 genes was significantly suppressed. However, the extent of variations in the expression of genes, except for FOLH1, was low (1.5-2.0). No significant variations were observed in the expression of the thymidylate synthase (TS), dihydropyrimidine dehydrogenase (DPD), thymidine phos- 
phorylase (TP), or orotate phosphoribosyltransferase (OPRT) genes, which were shown to be related to chemosensitivity to 5 -FU by several previous studies.

In the present study, the resistant tumor group (A549) and moderately effective group (Lu99, Lu130 and LC6) were classified by the TGI rate of S-1. We identified genes that were correlated with sensitivity to S-1. The expression of the $\mathrm{ABCC} 1,2$ and TST genes was negatively correlated with TGI, while the expression of the TK1 and ERCC2 genes was positively correlated with TGI.

ATP-binding cassette (ABC) transporters are a superfamily of membrane proteins that are best known for their ability to transport a wide variety of exogenous and endogenous substances across membranes against a concentration gradient via ATP hydrolysis. Seven subfamilies of human $\mathrm{ABC}$ transporters, one of the largest being the ' $\mathrm{C}$ ' subfamily (gene symbol $\mathrm{ABCC}$ ), have so far been identified (13). In tumor cells, $\mathrm{ABCC} 1$ can confer resistance to not only widely used antineoplastic drugs, including doxorubicin, methotrexate (MTX), daunorubicin, vincristine, and etoposide (14-17), but also some of the so-called 'targeted' agents (e.g., certain tyrosine kinase inhibitors) (18). ABCC1 was previously shown to be highly expressed in leukemias, esophageal carcinomas, and non-small cell lung cancer (19). Previous studies reported that $\mathrm{ABCC} 1$ might play a role in clinical chemotherapy resistance in breast cancer and as a predictor of poor prognosis in breast cancer patients receiving chemotherapy as a first-line systemic treatment for recurrence (20). However, the overall contribution of this drug transporter to clinical drug resistance has not yet been fully established (21).

Although the function of $\mathrm{ABCC} 2$ is similar to that of $\mathrm{ABCC} 1$, the tissue distribution of their expression is different. ABCC2 transports various anticancer drugs, including MTX, cisplatin, irinotecan, paclitaxel, and vincristine, in tumor cells (22-24). ABCC2 is expressed in several solid tumors such as renal carcinomas, colon carcinomas, breast carcinomas, lung carcinomas, and acute myelogenous leukemia $(25,26)$. Korita et al reported that $\mathrm{ABCC} 2$ overexpression correlated with a lower percentage of tumor necrosis in patients treated with cisplatin-based neoadjuvant chemotherapy for hepatocellular carcinoma (27). Our results suggest that ABCC transporter proteins may contribute to drug resistance to S-1.

Rhodanese is a mitochondrial enzyme that comprises two isoenzymes: thiosulfate sulfurtransferase (TST) and mercaptopyruvate sulfurtransferase (MST). The product of the TST gene is thiosulfate sulfurtransferase. Rhodanese can play a central role in detoxifying hydrogen sulfide (H2S) (28). Ramasamy et al reported that dysregulation in TST expression and activity results in the inability to effectively detoxify, and this could be a factor in the cell loss and inflammation that accompany ulcerative colitis and, ultimately, colorectal cancer (29). Although the relationship between TST and resistance of chemotherapy has not yet been determined, Ooyama et al demonstrated that TST gene expression was correlated with the sensitivity of 30 human tumor xenografts to $S-1$ (30).

Thymidine kinase (TK) and thymidylate synthase (TS) are key enzymes for pyrimidine synthesis, which is necessary for DNA synthesis. TK catalyzes the phosphorylation of thymidine for the salvage synthesis of deoxythymidine monophosphate (dTMP) (31). TK-1 is a cytosolic isozyme that is associated with the cell cycle. A strong correlation has been reported between TK activity and the amount of TK-1 protein or mRNA during the cell cycle $(32,33)$. However, there is disagreement among various investigators concerning the relationship between TK-1 activity or mRNA in human cancer cell lines and resistance to fluoropyrimidines $(8,34)$.

The ERCC2 (XPD) protein plays a role in DNA repair, particularly the nucleotide excision repair (NER) pathway, which recognizes and repairs a wide range of structurally unrelated lesions such as bulky adducts and thymidine dimers. Two ERCC2 polymorphisms, Asp312Asn (db SNP no. rs1799793) and Lys751Gln (db SNP no. rs13181), have mainly been investigated. These SNPs may modulate the repair capacity and contribute to individual variations in the response to chemotherapy (35). Although relationships between ERCC2 SNPs and platinum activity have been reported in patients with esophageal, gastric, colorectal and NSCLC (36-38), the association between ERCC 2 and treatment outcomes in solid tumors treated with platinum-based chemotherapy remains controversial.

We used a low density array representing 93 genes to analyze expression profiles in 4 orthotopically implanted lung cancers derived from human lung cancer cell lines in an attempt to identify genes that may be associated with chemosensitivity to the antitumor drug S-1. We demonstrated that the expression of ABCC1, 2, and TST genes was negatively correlated with TGI, while the expression of the TK1 and ERCC2 genes was positively correlated with TGI. Further studies to examine the relationship between the expression of these genes and resistance to the antitumor drug S-1 are warranted.

\section{Acknowledgements}

Kazuya Kondo and Akira Tangoku have received research funding from Taiho Pharmaceutical Co., Ltd. Etsuko Sakamoto and Junji Uchida are employees of Taiho Pharmaceutical Co., Ltd.

\section{References}

1. Zembutsu H, Ohnishi Y, Tsunoda T, et al: Genome-wide cDNA microarray screening to correlate gene expression profiles with sensitivity of 85 human cancer xenografts to anticancer drugs. Cancer Res 62: 518-527, 2002.

2. Dan S, Tsunoda T, Kitahara O, et al: An integrated database of chemosensitivity to 55 anticancer drugs and gene expression profiles of 39 human cancer cell lines. Cancer Res 62: 1139-1147, 2002.

3. Park JS, Young Yoon S, Kim JM, Yeom YI, Kim YS and Kim NS: Identification of novel genes associated with the response to 5-FU treatment in gastric cancer cell lines using a cDNA microarray. Cancer Lett 214: 19-33, 2004.

4. Fukushima M, Fujioka A, Uchida J, Nakagawa F and Takechi T: Thymidylate synthase (TS) and ribonucleotide reductase (RNR) may be involved in acquired resistance to 5-fluorouracil (5-FU) in human cancer xenografts in vivo. Eur J Cancer 37: 1681-1687, 2001.

5. Francia G, Cruz-Munoz W, Man S, Xu P and Kerbel RS: Mouse models of advanced spontaneous metastasis for experimental therapeutics. Nat Rev Cancer 11: 135-141, 2011.

6. Paget $S$ : The distribution of secondary growths in cancer of the breast. Lancet 133: 571-573, 1889.

7. Tashiro T, Inaba M, Kobayashi T, et al: Responsiveness of human lung cancer/nude mouse to antitumor agents in a model using clinically equivalent doses. Cancer Chemother Pharmacol 24: 187-192, 1989. 
8. Takechi T, Okabe H, Ikeda K, et al: Correlations between antitumor activities of fluoropyrimidines and DPD activity in lung tumor xenografts. Oncol Rep 14: 33-39, 2005.

9. Ishikura H, Kondo K, Miyoshi T, Kinoshita H, Hirose T and Monden Y: Artificial lymphogenous metastatic model using orthotopic implantation of human lung cancer. Ann Thorac Surg 69: 1691-1695, 2000.

10. Fujino H, Kondo K, Ishikura H, et al: Matrix metalloproteinase inhibitor MMI-166 inhibits lymphogenous metastasis in an orthotopically implanted model of lung cancer. Mol Cancer Ther 9: 1409-1416, 2005.

11. Kondo K, Fujino H, Miyoshi T, Ishikura H, Sakiyama S and Monden Y: Orthotopically implanted SCID mouse model of human lung cancer suitable for investigating metastatic potential and anticancer drug effects. Oncol Rep 5: 991-999, 2004.

12. Fujino H, Kondo K, Miyoshi T, et al: Establishment of patientlike SCID mouse model by orthotopically implanting primary cultured cells from surgically-resected lung cancer tissues. Oncol Rep 6: 1709-1715, 2003

13. Chen ZS and Tiwari AK: Multidrug resistance proteins (MRPs/ABCCs) in cancer chemotherapy and genetic diseases. FEBS J 278: 3226-3245, 2011.

14. Borst P, Evers R, Kool M and Wijnholds J: A family of drug transporters: the multidrug resistance associated proteins. J Nat Cancer Inst 92: 1295-1302, 2000.

15. Loe DW, Almquist KC, Deeley RG and Cole SP: Multidrug resistance protein (MRP)-mediated transport of leukotriene C4 and chemotherapeutic agents in membrane vesicles. Demonstration of glutathione-dependent vincristine transport. J Biol Chem 271: 9675-9682, 1996.

16. Tiwari AK, Sodani K, Dai CL, Ashby CR Jr and Chen ZS Revisiting the $\mathrm{ABCs}$ of multidrug resistance in cancer chemotherapy. Curr Pharm Biotechnol 12: 570-594, 2011.

17. Loe DW, Deeley RG and Cole SP: Characterization of vincristine transport by the M(r) 190,000 multidrug resistance protein (MRP): evidence for cotransport with reduced glutathione. Cancer Res 58: 5130-5136, 1998.

18. Hegedus T, Orfi L, Seprodi A, Varadi A, Sarkadi B and Keri G: Interaction of tyrosine kinase inhibitors with the human multidrug transporter proteins, MDR1 and MRP1. Biochim Biophys Acta 1587: 318-325, 2002.

19. Nooter K, Westerman AM, Flens, MJ et al: Expression of the multidrug resistance-associated protein (MRP) gene in human cancers. Clin Cancer Res 1: 1301-1310, 1995.

20. Nooter K, Brutel de la Riviere G, Klijn J, Stoter G and Foekens J: Multidrug resistance protein in recurrent breast cancer. Lancet 349: 1885-1886, 1997.

21. Deeley RG, Westlake C and Cole SP: Transmembrane transport of endo- and xenobiotics by mammalian ATP-binding cassette multidrug resistance proteins. Physiol Rev 86: 849-899, 2006.

22. Cui Y, Konig J, Buchholz JK, Spring H, Leier I and Keppler D: Drug resistance and ATP-dependent conjugate transport mediated by the apical multidrug resistance protein, MRP2, permanently expressed in human and canine cells. Mol Pharmacol 55: 929-937, 1999.

23. Chu XY, Kato Y, Niinuma K, Sudo KI, Hakusui $H$ and Sugiyama Y: Multispecific organic anion transporter is responsible for the biliary excretion of the camptothecin derivative irinotecan and its metabolites in rats. J Pharmacol Exp Ther 281: 304-314, 1997
24. Huisman MT, Chhatta AA, van Tellingen O, Beijnen JH and Schinkel AH: MRP2 (ABCC2) transports taxanes and confers paclitaxel resistance and both processes are stimulated by probenecid. Int J Cancer 116: 824-829, 2005.

25. Sandusky GE, Mintze KS, Pratt SE and Dantzig AH: Expression of multidrug resistance-associated protein 2 (MRP2) in normal human tissues and carcinomas using tissue microarrays. Histopathology 41: 65-74, 2002.

26. van der Kolk DM, de Vries EG, Koning JA, van den Berg E, Muller $M$ and Vellenga E: Activity and expression of the multidrug resistance proteins MRP1 and MRP2 in acute myeloid leukemia cells, tumor cell lines, and normal hematopoietic $\mathrm{CD}^{+}{ }^{+}$peripheral blood cells. Clin Cancer Res 4: 1727-1736, 1998.

27. Korita PV, Wakai T, Shirai Y, et al: Multidrug resistance associated protein 2 determines the efficacy of cisplatin in patients with hepatocellular carcinoma. Oncol Rep 23: 965-972, 2010.

28. Nagahara $\mathrm{N}$ and Nishino T: Role of amino acid residues in the active site of rat liver mercaptopyruvate sulfurtransferase. cDNA cloning, overexpression, and site-directed mutagenesis. J Biol Chem 271: 27395-27401, 1996.

29. Ramasamy S, Singh S, Taniere P, Langman MJ and Eggo MC: Sulfide-detoxifying enzymes in the human colon are decreased in cancer and upregulated in differentiation. Am J Physiol Gastrointest Liver Physiol 291: G288-G296, 2006.

30. Ooyama A, Takechi T, Toda E, et al: Gene expression analysis using human cancer xenografts to identify novel predictive marker. Cancer Sci 97: 510-522, 2006

31. Golub TR, Slonim DK, Tamayo P, et al: Molecular classification of cancer: class discovery and class prediction by gene expression monitoring. Science 286: 531-537, 1999.

32. Golub TR: Genome-wide views of cancer. N Engl J Med 8: 601-602, 2001.

33. Mayer F, Hartmann JT, von Pawel J, et al: A phase I study of oral uracil-ftorafur plus folinic acid in combination with weekly paclitaxel in patients with solid tumors. Ann Oncol 5: 755-759, 2002.

34. Nukatsuka M, Fujioka A, Nakagawa F, et al: Antimetastatic and anticancer activity of S-1, a new oral dihydropyrimidine-dehydrogenase inhibiting fluoropyrimidine, alone and in combination with paclitaxel in an orthotopically implanted human breast cancer model. Int J Oncol 25: 1531-1536, 2004.

35. Kiyohara $\mathrm{C}$ and Yoshimasu K: Genetic polymorphisms in the nucleotide excision repair pathway and lung cancer risk: a metaanalysis. Int J Med Sci 4: 59-71, 2007.

36. Ott K, Rachakonda PS, Panzram B, et al: DNA repair gene and MTHFR gene polymorphisms as prognostic markers in locally advanced adenocarcinoma of the esophagus or stomach treated with cisplatin and 5-fluorouracil-based neoadjuvant chemotherapy. Ann Surg Oncol 18: 2688-2698, 2011.

37. Yin M, Yan J, Martinez-Balibrea E, et al: ERCC1 and ERCC2 polymorphisms predict clinical outcomes of oxaliplatin-based chemotherapies in gastric and colorectal cancer: a systemic review and metaanalysis. Clin Cancer Res 17: 1632-1640, 2011.

38. Gurubhagavatula S, Liu G, Park S, et al: XPD and XRCC1 genetic polymorphisms are prognostic factors in advanced non-small cell lung cancer patients treated with platinum chemotherapy. J Clin Oncol 22: 2594-2601, 2004. 